\title{
Cas clinique : Intoxication mortelle par le dinitro-orthocresol
}

\author{
Case report : \\ Dinitro-orthocresol fatal intoxication
}

\section{Sabrina BOUANANI(1), Jean-Michel GAULIER ${ }^{(1)}$, Sylvie CALVAT ${ }^{(2)}$, Sylvain DULAURENT( ${ }^{(1)}$, Anissa CHACHIA ${ }^{(3)}$, Patrick HARRY ${ }^{(4)}$, Gérard LACHATRE(1)}

(1) Service de Pharmacologie et Toxicologie, CHU Dupuytren, Limoges

(2) Service de Réanimation,

(3) Laboratoire de Biologie, Centre Hospitalier, Angoulême

(4) Centre Antipoison, Angers

*Auteur à qui adresser la correspondance : J-M GAULIER, Service de Pharmacologie et Toxicologie,

2, avenue Martin-Luther-King, CHU Dupuytren, 87042 Limoges Cedex Tel : 33 (0)5 55056140 Fax : 33 (0)5 55056162 e-mail : jm-gaulier@unilin.fr

(Article reçu le 6 juin 2006 ; accepté après modifications le 30 septembre 2006)

\section{RÉSUMÉ}

Une femme de 56 ans a été admise à l'hôpital après ingestion d'un liquide inconnu. Consciente et polypnéique, elle présentait une pigmentation péri-buccale jaune. Son état clinique s'est aggravé très rapidement (agitation, cyanose, puis contracture généralisée) pour aboutir au décès. Du dinitro-orthocrésol (DNOC) à été mis en évidence par chromatographie liquide couplée à la spectrophotométrie $U V$ à barrette de diodes dans le liquide gastrique, le sang $(34 \mathrm{mg} / L)$ et les urines. Le mode d'action de cet herbicide sélectif interdit en France depuis 2000 repose sur le découplage de la phosphorylation oxydative et le traitement des intoxications parfois mortelles ne peut être que symptomatique.

\section{MOTS-CLÉS}

Dinitro-orthocrésol, herbicide, intoxication, concentration sanguine.

\section{SUMMARY}

A 56 year old woman was admitted to hospital after ingestion of an unknown liquid. Conscious with polypnoea, a yellow pigmentation was observed around her mouth. Her clinical state declined rapidly (agitation, cyanosis and muscular contractions) and finally resulted in her decease. Dinitro-orthocrésol (DNOC) was highlighted by liquid chromatography coupled with a diode-array UV detector in the gastric liquid, blood ( $34 \mathrm{mg} / \mathrm{L})$ and urines. The action of this selective herbicide prohibited in France since 2000 is based on the decoupling of oxidative phosphorylation and the treatment of the intoxication, sometimes fatal, can only be symptomatic.

\section{KEY-WORDS}

Dinitro-ortho-cresol, herbicide, intoxication, blood concentration. 
L'utilisation des herbicides s'est aujourd'hui généralisée, voire banalisée en France. L'usage des dinitrophénols (ou « huiles jaunes »), phénols substitués, bien qu'interdit depuis 2000 peut encore être à l'origine d'intoxications. Nous présentons un cas de décès lié à l'absorption accidentelle de dinitro-orthocrésol (DNOC), produit de base des dinitrophénols et chef de file des découplants de la phosphorylation oxydative.

\section{Description du cas}

Une femme de 56 ans, éthylique chronique, a ingéré par mégarde un liquide inconnu. A son admission (H1), la patiente est encore consciente : TA $199 / 82 \mathrm{mmHg}$, FC $130 / \mathrm{min}, \mathrm{FR} 35 / \mathrm{min}, \mathrm{SpO} 2=100 \%$, TC $36^{\circ} \mathrm{C}$. Sur le plan ventilatoire, il apparaît une polypnée ample (auscultation pulmonaire et radio normale) associée à une acidose lactique isolée ( $9 \mathrm{mM})$. La patiente exprime une intense sensation de chaleur, avec hypersudation profuse. Les conjonctives sont jaunes alors que la bilirubine est normale.

A son transfert en réanimation (H2), la patiente est agitée et présente une cyanose périphérique, puis une contracture musculaire généralisée extrême, résistante aux curares, avec une alternance de tachycardie ventriculaire et d'asystolie associée à un œdème pulmonaire conduisant rapidement au décès (H5). Du contenu gastrique, du sang et des urines ont été prélevés pour analyses toxicologiques.

\section{Matériel et méthode}

Une recherche large de médicaments et toxiques a été effectuée dans le contenu gastrique par chromatographie liquide couplée à la spectrophotométrie UV à barrette de diodes (CL/UV-BD), couplée à spectrométrie de masse en tandem (CL-SM/SM), et par chromatographie gazeuse couplée à la spectrométrie de masse (CG/SM). Des recherches spécifiques de glycols et de solvants volatils ont été réalisées dans ce même milieu respectivement par CG/SM et Headspace-CG/SM.

A la suite de sa mise en évidence dans le liquide gastrique lors du screening par CL/UV-BD, un dosage de DNOC a été réalisé dans les trois milieux biologiques par CL/UV-BD (LC-10, Shimadzu, France) : l'extraction a été réalisée après ajout de l'étalon interne (N-méthylclonazepam) à $500 \mu \mathrm{L}$ de prise d'essai par un mélange chloroforme/propanol-2/n-heptane (60/14/26, $\mathrm{v} / \mathrm{v} / \mathrm{v}$ ) en milieu alcalin [tampon $\mathrm{NH}_{4} \mathrm{Cl}$ saturé/eau désionisée $\left(30 / 70\right.$, v/v) ajusté à $\mathrm{pH} 9,50$ avec $\mathrm{NH}_{4} \mathrm{OH}$ $25 \%$ ]. Après évaporation de la phase organique, le résidu a été repris par $100 \mu \mathrm{L}$ d'un mélange de tampon phosphate $0,025 \mathrm{M}$ à pH 2,60/acétonitrile (70/30, v/v). La détection a été réalisée à $270 \mathrm{~nm}$ après séparation chromatographique ( $20 \mu \mathrm{L}$ d'extrait injecté) sur colonne Nucléosil C18 par un gradient de tampon phosphate à $\mathrm{pH}$ 2,60/acétonitrile. Le dosage urinaire a été réalisé avec, et sans, étape d'hydrolyse préalable (Béta-glucuronidase à $37^{\circ} \mathrm{C}$ pendant 12 heures). Un exemple de chromatogramme obtenu est présenté en figure 1. Les limites de détection et de quantification étaient respectivement de 0,05 et $0,1 \mathrm{mg} / \mathrm{L}$.

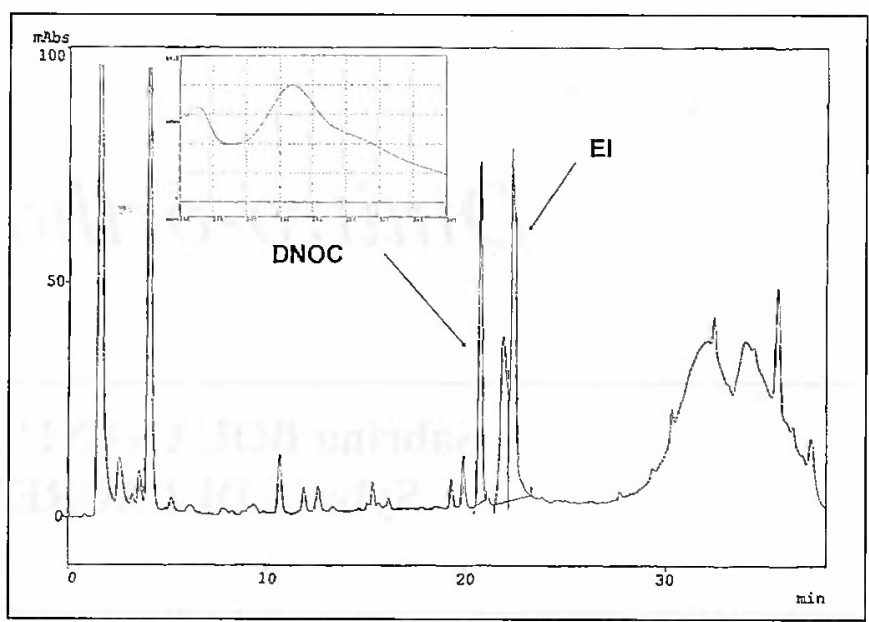

Figure 1: Chromatogrannme à 270 nm de l'extrait urinaire de la patiente (après hydrolyse).

\section{Résultats et discussion}

Des hydrocarbures aromatiques étaient présents dans le contenu gastrique et đu DNOC a été décelé et quantifié dans les 3 milieux biologiques (Tableau I).

Contrairement aux crésols qui sont des désinfectants, le DNOC est un herbicide sélectif qui était encore utilisé à la fin du siècle dernier en traitement hivernal des céréales, de la vigne et des arbres fruitiers. Les formulations qui comprenaient régulièrement des dérivés du pétrole peuvent expliquer la présence d'hydrocarbures dans le contenu gastrique.

Tableau I : résultat des dosages de DNOC dans les échantillons biologiques mis à notre disposition.

\begin{tabular}{|c|c|c|c|}
\hline & $\begin{array}{l}\text { Contenu } \\
\text { gastrique }\end{array}$ & Sang total & Urine \\
\hline $\begin{array}{l}\text { DNOC } \\
(\mathrm{mg} / \mathrm{L})\end{array}$ & 119 & 34 & $\begin{array}{c}3,3 \text { (forme non conjuguée) } \\
5,7 \text { (forme totale) }\end{array}$ \\
\hline
\end{tabular}

Le DNOC, qui est par ailleurs un produit mutagène, a été à l'origine de nombreuses intoxications accidentelles, parfois mortelles, à la suite d'ingestions, d'expositions cutanées ou pulmonaires. Toutefois, les données de la littérature sont rares, et surtout très anciennes (1-4). 
Le DNOC s'élimine lentement et approximativement $2 \%$ de la dose ingérée est excrété au cours des premières $24 \mathrm{~h}$ sous forme inchangée dans les urines (3). Au total, c'est 4 à $10 \%$ du produit qui sont éliminés sous forme inchangée dans les urines, le métabolisme conduisant pour l'essentiel à des dérivés amino- qui sont éliminés sous forme conjuguée. Des concentrations sanguines de l'ordre de $20 \mathrm{mg} / \mathrm{L}$ pourraient être associées à l'apparition d'une toxicité, et une concentration sanguine de $75 \mathrm{mg} / \mathrm{L}$ a été mesurée à la suite d'un décès $(2,3)$.

Le mode d'action de ce toxique à effet cumulatif, repose sur le découplage des phosphorylations oxydatives (5). Ce découplage enzymatique avec rupture de la force proton-motrice et conversion de l'énergie en chaleur explique l'hyperthermie cliniquement observée. Les signes d'intoxication aiguë apparaissent rapidement : coloration jaune des muqueuses et de la peau, hyperthermie, sudation, vomissements, déshydratation, polydipsie, polypnée, oligurie, coma, convulsions. Sur le plan neurologique, il est régulièrement rapporté des sensations d'euphorie et de vertiges, suivies d'état d'agitation, d'anxiété et de confusion mentale. Les effets toxiques observés ici (polypnée, coma, convulsion, arythmies cardiaques et œdème pulmonaire) peuvent être suivis d'une atteinte hépatique et rénale plus tardive, et le traitement ne peut être que symptomatique (6).

\section{Références}

1. Batchelor G.S., Walker K.C., Elliot J.W. Dinitroorthocresol exposure from apple-thinning sprays. Arch. Ind. Health $1956 ; 13: 593-6$.

2. Harvey D.G., Bidstrup P.L., Bonnell J.A.L. Poisoning by dinitro-ortho-cresol. Some observations on the effects of dinitro-ortho-cresol administred by mouth to human votunteers. Brit.Med. $1951 ; 213-6$.

3. Bidstrup P.L., Payne D.J.H. Poisoning by dinitroortho-cresol. Brit. Med. J. 1951 ; 2 : 16-9.

4. Harvey D.G., Bidstrup P.L., Bonnell J.A.L. Prevention of acute dinitro-ortho-cresol (DNOC) poisoning. Lancet $1952 ; 1: 794-5$.

5. Castilho R.F., Vicente J.A., Kowaltowski A.J., Vercesi A.E. 4,6-Dinitro-o-cresol uncouples oxidative phosphorylation and induces membrane permeability transition in rat liver mitochondria. Int. J. Biochem. Cell. Biol. 1997 ; 29(7) : 1005-11.

6. Flesch F., Tournoud C., Saviuc P. Intoxication par herbicides. In : Danel V., Barriot P. ed. Intoxications aiguës en réanimation. Rueil-Malmaison : Arnette, $1999: 236-7$. 